\title{
Leucine rich repeat LGI family member 3: Integrative analyses reveal its prognostic association with non-small cell lung cancer
}

\author{
DONG-SEOK KIM, NYOUN SOO KWON and HYE-YOUNG YUN
}

\author{
Department of Biochemistry, Chung-Ang University, College of Medicine, Seoul 06974, Republic of Korea
}

Received December 31, 2018; Accepted June 21, 2019

DOI: $10.3892 / \mathrm{ol} .2019 .10648$

\begin{abstract}
Leucine rich repeat LGI family member 3 (LGI3) is a member of the LGI protein family. Our previous studies reported that LGI3 was expressed in adipose tissues, brain and skin, where it served roles as a multifunctional cytokine and pro-inflammatory adipokine. It was hypothesized that LGI3 may be involved in cytokine networks in cancer. The present study aimed to analyze differentially expressed genes in non-small cell lung cancer (NSCLC) tissues and NSCLC cohort data, to evaluate the prognostic role of LGI3. Expression microarray and NSCLC cohort data were statistically analyzed by bioinformatic methods, and protein-protein interactions, functional enrichment and pathway, gene coexpression network (GCN) and prognostic association analyses were performed. The results demonstrated that the expression levels of LGI3 and its receptor a disintegrin and metalloproteinase domain-containing protein 22 were significantly decreased in NSCLC tissues. A total of two upregulated genes and 11 downregulated genes in NSCLC tissues were identified as LGI3-regulated genes. Protein-protein interaction network analysis demonstrated that all LGI3-regulated genes that were altered in NSCLC were involved in a protein-protein interaction network cluster. Functional enrichment, Kyoto Encyclopedia of Genes and Genomes pathway and GCN analyses demonstrated the association of these genes with the immune and inflammatory responses, angiogenesis, the tumor necrosis factor pathway, and chemokine and peroxisome proliferator-activated receptor signaling pathways. Analysis of NSCLC cohorts revealed that low expression levels of LGI3 was significantly associated with poor prognosis of NSCLC. Analysis of the somatic mutations of the LGI3 gene in NSCLC revealed that the amino acid residues altered in NSCLC included two single nucleotide polymorphism sites and three phylogenetically coevolved amino acid residues.
\end{abstract}

Correspondence to: Dr Hye-Young Yun, Department of Biochemistry, Chung-Ang University, College of Medicine, 84 Heukseok-ro, Dongjak, Seoul 06974, Republic of Korea

E-mail: hyyunoffice@gmail.com

Key words: leucine rich repeat LGI family member 3, cytokine, adipokine, non-small cell lung cancer, network, prognosis
Taken together, these results suggest that LGI3 may be a potential prognostic marker of NSCLC.

\section{Introduction}

Leucine rich repeat LGI family member 3 (LGI3; formerly known as leucine-rich glioma inactivated 3) is a secretory protein member of the LGI family in vertebrates that has been identified to be highly expressed in the brain (1). LGI3 expression in the brain has been reported to be regulated at the transcription level by activating enhancer-binding protein 2 (AP-2) and neuronal restrictive silencer (1). Our previous studies demonstrated that LGI3 regulates exocytosis and the differentiation of neuronal cells $(2,3)$. LGI3 is expressed in the epidermal layer of the skin where it may act as a cutaneous cytokine (4). Our previous study found that LGI3 is secreted in response to ultraviolet $\mathrm{B}$ irradiation and protects keratinocytes (4). Additionally, we previously reported that LGI3 increases migration and differentiation of keratinocytes $(5,6)$ and melanocyte pigmentation (7).

Our previous studies revealed that LGI3 is expressed in adipose tissues and its expression is downregulated during adipogenesis and upregulated in adipose tissues in obesity $(8,9)$. Furthermore, one of our previous studies demonstrated that LGI3 attenuates adipogenesis through a disintegrin and metalloproteinase domain-containing protein (ADAM)23, which is one of the receptors for LGI3 (ADAM22 and ADAM23), and that LGI3 increases pro-inflammatory genes, including tumor necrosis factor- $\alpha$ (TNF- $\alpha$ ) in macrophage cells (9). LGI3 negatively regulates adiponectin (8). LGI3 and TNF- $\alpha$ increase expression mutually through NF- $\mathrm{kB}$, suggesting their positive cooperativity in promoting metabolic inflammation in obesity (10). We postulated that LGI3 is a pleiotropic cytokine and adipokine secreted by and acting at multiple cell types, and that LGI3 may be a pro-inflammatory cytokine that interacts with various cytokines, adipokines, chemokines and signaling proteins (11).

Our recent studies proposed that LGI3 may be involved in the cytokine network in cancer (11-13). Our recent study reported that LGI3 expression is associated with the prognosis of glioma (13). Furthermore, the expression levels and genetic variations of LGI3 may have potential prognostic roles in various types of cancer (12). The present study utilized integrative analyses of gene expression microarrays, gene product networks and patient cohorts, and presented evidence 
supporting the potential prognostic role of LGI3 in non-small cell lung cancer (NSCLC), which is the most common type of lung cancer affecting $85 \%$ of patients (14).

\section{Materials and methods}

Gene expression microarray data. The mRNA expression microarray dataset was retrieved from the Gene Expression Omnibus database (http://www.ncbi.nlm.nih.gov/geo/). The dataset GSE19804 is based on the GPL570 [HG-U133 Plus_2] Affymetrix Human Genome U133 Plus 2.0 Array (Affymetrix; Thermo Fisher Scientific, Inc.) platform (15). The dataset contained 120 samples, including 60 samples of NSCLC tissues and 60 samples of paired adjacent normal lung tissues from nonsmoking patients with NSCLC.

Data processing for identification of differentially expressed genes (DEGs). The microarray data were analyzed using the affy package (version 1.62.0) in $\mathrm{R} 3.5 .1$ (http://www.r-project.org) (16). The dataset was subjected to background correction, quantile normalization and probe summarization of expression values. The $\log 2$ intensities of the probeset were calculated by the Robust Multichip Average algorithm of the affy package (16). Gene expression data were averaged to provide the final expression values for multiple probes for the same gene symbols. Probesets to non-expressed mRNAs were excluded using the Affymetrix Microarray Suite 5 calls algorithm (version 5.0; web.mit. edu/ r/current/arch/i386_linux26/lib/R/library/affy/html/mas5calls. html). Differential expression analysis was conducted using the limma package (version 3.40.2; bioconductor.org/ packages/release/bioc/html/limma.html) in R 3.5.1. Genes with $\mathrm{P}<0.05$ and $\log _{2}$ (fold change) $\mid \geq 1$ were considered to be statistically significant DEGs.

Comparative analysis, protein-protein interaction network, and functional enrichment and gene coexpression network $(G C N)$ analyses of DEGs. Comparative analysis of categorized gene groups was presented using a Venn diagram generated by Venny 2.1 (bioinfogp.cnb.csic.es/tools/venny). The protein-protein interaction network was generated using data from the Search Tool for the Retrieval of Interacting Genes (version 10.5; string-db.org) (17), and was visualized by Cytoscape 3.7.0 using an interaction degree-sorted circle layout (18). Functional enrichment analysis and Kyoto Encyclopedia of Genes and Genomes (KEGG) pathway analysis were performed using the Database for Annotation, Visualization and Integration Discovery (version 6.8; david. abcc.ncifcrf.gov) (19). Results were sorted by P-values and the subsets of the entries with $\mathrm{P}<0.05$ were presented. GCN analysis was performed using the GCN of human lung (uuid: $0 \mathrm{c} 14 \mathrm{bd} 7$ 0-5cae-11e7-8f50-0ac135e8bacf) (20) from The Network Data Exchange database (version 2.3.1; www.ndexbio.org) and visualized by Cytoscape 3.7.0 using the prefuse force directed layout. Gene Ontology (GO) terms of the GCN were mapped by BiNGO (version 3.0.3; apps.cytoscape.org/apps/bingo) and visualized by Cytoscape 3.7.0 using a hierarchical layout. Transcriptional regulatory associations between transcription factors and the groups of genes were analyzed by Transcription Factor Affinity Prediction tools (trap.molgen.mpg.de) (21).
Meta-analysis of patient cohorts. The datasets of gene expression microarray analysis for NSCLC cohorts were retrieved from the Prognoscan database (http://www.prognoscan.org; Table III) (22). Datasets in the Prognoscan database were previously processed through quality control tests, normalization and batch effect adjustment and the exclusion of low-quality samples. The association between gene expression values and NSCLC prognosis was assessed by the minimum P-value method for survival analysis of patient groups that calculates the cut-point in a continuous gene expression measurement. Patients ranked by gene expression values were divided at the cut-off point to minimize the P-value, and the difference of survival between high and low gene expression groups was calculated using a log-rank test. The statistically significant $(\mathrm{P}<0.05)$ datasets were used to generate Kaplan-Meier plots.

Somatic mutations in NSCLC. Somatic mutations of the LGI3 gene in NSCLC were identified in Cbioportal (www.cbioportal. org), the Catalogue of Somatic Mutations in Cancer (cancer. sanger.ac.uk/cosmic) and The Cancer Genome Atlas (hportal. gdc.cancer.gov). Data of conserved residues, phylogenetically coevolved residues and single nucleotide polymorphisms (SNPs) of LGI3 have been previously described (12). A Venn diagram of the categorized genetic variations was generated using InteractiVenn (www.interactivenn.net).

\section{Results}

Differential expression of LGI3 in NSCLC. Our previous study reported that somatic mutations and expression of LGI3 may have prognostic significance in various types of cancer (12). Therefore, it was hypothesized that LGI3 may be associated with the cytokine network in lung cancer. In the present study, analysis of DEGs in the lung tissues from patients with NSCLC revealed that LGI3 expression was significantly lower than in normal tissues (fold change, $0.41 ; \mathrm{P}=8.43 \times 10^{-20}$; Fig. 1). The expression levels of the LGI3 receptor ADAM22 were significantly decreased in NSCLC tissues (fold change, 0.72; $\mathrm{P}=7.01 \times 10^{-8}$; Fig. 1). Expression of ADAM23 was not significantly changed in NSCLC tissues (Fig. 1).

Identification of LGI3-regulated and NSCLC-altered genes, and their protein-protein interaction network. Analysis of DEGs in the expression microarray dataset of NSCLC revealed that 1,158 genes were upregulated and 1,526 genes were downregulated in NSCLC tissues ( $\mid \log _{2}$ (fold change) $\mid \geq 1$ and $\mathrm{P}<0.05$ ). Our previous study identified 48 gene products that are regulated by LGI3 [adiponectin (ADIPOQ), Akt1, BAD, complement C5 (C5), C-C motif chemokine ligand (CCL)2, CCL12, CD68, CCAAT enhance binding protein $\alpha$, C-reactive protein (CRP), colony stimulating factor (CSF)1, $\mathrm{CSF} 3$, catenin $\beta 1, \mathrm{C}-\mathrm{X}-\mathrm{C}$ motif chemokine ligand (CXCL)2, CXCL13, cytochrome b-24 $\alpha$ chain, cytochrome b-24 $\beta$ chain, $\delta$ like non-canonical Notch ligand 1 (DLK1), eukaryotic translation initiation factor $4 \mathrm{E}$ binding protein 1 , adhesion $\mathrm{G}$ protein-coupled receptor E1, endothelial cell specific molecule 1 (ESM1), fatty acid binding protein 4 (FABP4), glycogen synthase kinase 3 (GSK3)A, GSK3B, insulin like growth factor 1 (IGF1), IGF binding protein (IGFBP)1, IGFBP5, interleukin 6 (IL6), integrin subunit $\alpha$ X, lipoprotein lipase (LPL), 


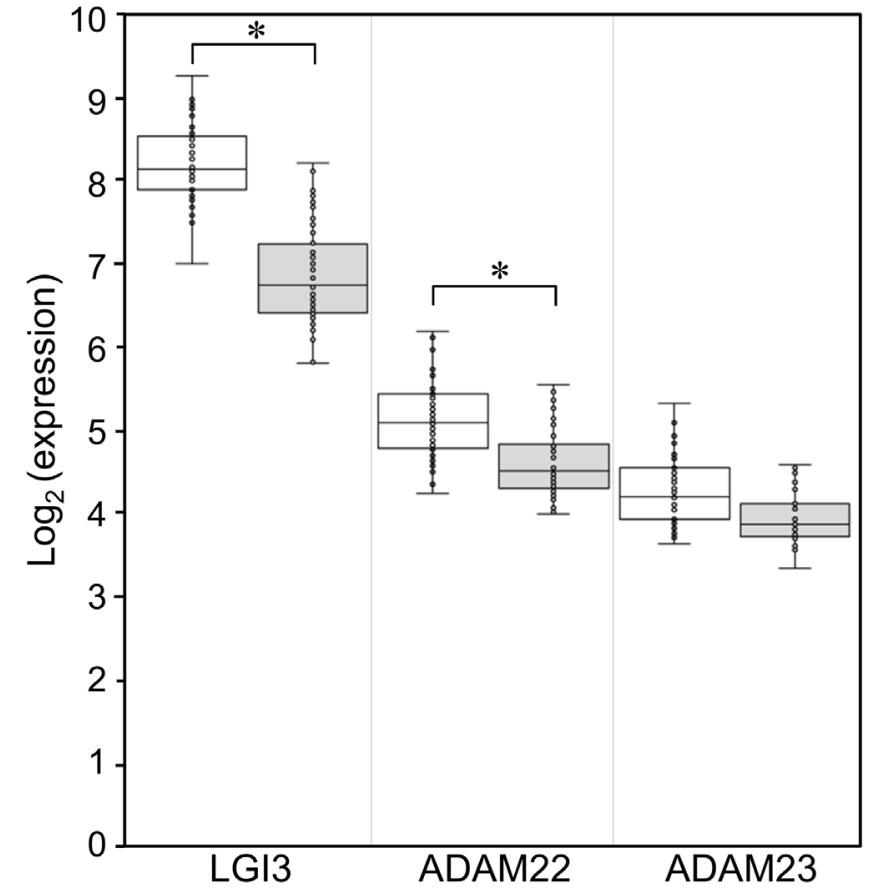

Figure 1. Differential expression of LGI3 and its receptors in NSCLC tissues. Control (non-tumor) tissues are shown in white. NSCLC tissues are shown in grey. ${ }^{*} \mathrm{P}<0.001$. ADAM, a disintegrin and metalloproteinase domain-containing protein; LGI3, leucine rich repeat LGI family member 3; NSCLC, non-small cell lung cancer.

mitogen-activated protein kinase (MAPK)1, MAPK3, MDM2 proto-oncogene (MDM2), microphthalmia-associated transcription factor (MITF), neutrophil cytosolic factor (NCF)1, NCF2, NF- $\mathrm{KB} 1$, nitric oxide synthase 2 , PI3K catalytic subunit $\alpha$, peroxisome proliferator activated receptor $\gamma$ (PPARG), protein kinase AMP-activated catalytic subunit $\alpha 1$, PTEN, prostaglandin-endoperoxide synthase 2 (PTGS2), protein tyrosine kinase 2, serpin family E member 1 (SERPINE1), syntaxin 1A, TIMP metallopeptidase inhibitor 1 (TIMP1), TNF and tumor protein p53] (11). Venn diagram analysis of NSCLC-altered and LGI3-regulated genes demonstrated that one LGI3-upregulated gene and one LGI3-downregulated gene belonged to the set of NSCLC-upregulated genes, and that seven LGI3-upregulated genes and four LGI3-downregulated genes belonged to the set of NSCLC-downregulated genes (Fig. 2A). Overall, the expression levels of $27 \%(8 / 30)$ of the LGI3-upregulated genes and $28 \%(5 / 18)$ of the LGI3-downregulated genes were altered in NSCLC. Most of the LGI3-regulated gene products (94\%; $45 / 48$ genes) have been demonstrated to form a protein-protein interaction network cluster in our previous study (11). Protein-protein interaction network analysis of the 13 NSCLC-altered and LGI3-regulated genes demonstrated that all gene products formed an interaction network cluster (Fig. 2B). The proteins with the highest interaction degrees $(\geq 5)$ were CCL2 [also known as monocyte chemoattractant protein (MCP-1)], IL6, PPARG, SERPINE1, LPL and PTGS2 (also known as cyclooxygenase 2).

Functional enrichment and KEGG pathway analyses of LGI3-regulated and NSCLC-altered genes. The functional signature of LGI3-regulated genes that are altered in NSCLC tissues was investigated by functional enrichment analysis to obtain the GO terms of the gene groups (Table I). The genes were significantly associated with immune and inflammatory processes, including lipopolysaccharide response, chemokines, vascular endothelial growth factor receptor, cell redox homeostasis and cell response to tumor necrosis factor. Additionally, KEGG pathway analysis of LGI3-regulated and NSCLC-altered genes revealed that the genes were associated with the signaling pathways of TNF, chemokines, PPAR, and cytokines and infectious diseases (i.e. malaria and leishmaniasis; Table II). All associated KEGG pathways were closely associated with immune and inflammatory systems.

GCN analysis of LGI3-regulated and NSCLC-altered genes. To elucidate the roles of LGI3-regulated genes that are altered in NSCLC, this gene set was queried against the GCN of the lung. GCNs are a useful tool for exploring the roles of gene sets, since coexpressed genes are controlled by common transcriptional regulatory programs and are members of the same protein complex or signaling pathway (23). A total of 10 gene products in the gene set (Fig. 2B) were found in the lung GCN (group a; Fig. 3A) and these gene products were associated with 322 gene products in the network (groups $b$ and c; Fig. 3A). The subnetwork of coexpression, consisting of 322 gene products (Table SI), appeared to occupy a domain with two adjacent clusters in the GCN (groups b and c; Fig. 3A). A GO category map of the subnetwork (groups $\mathrm{b}$ and c; Fig. 3B) demonstrated that the gene products in the network were involved in immune (groups b and c) and inflammatory responses, including responses to wounding and stress (group c). Transcription factor affinity prediction of the genes in groups $b$ and $c$ suggested that these genes may be coexpressed under the common transcriptional regulatory processes by various immune and inflammatory transcription factors [Elf-1, ETS variant 4 (Pea3), Spi-1 proto-oncogene (Pu.1), C-ets-1, upstream transcription factor (Usf) 1/2, Stat6, $\mathrm{NF}-\kappa \mathrm{B}$ (Rela), cAMP response element-binding protein (CREB) and AP-2; Table SII].

Association of LGI3 with prognosis of NSCLC. Downregulation of LGI3 in NSCLC tissues (Fig. 1) suggested an association of LGI3 with the morbidity and mortality of NSCLC. To investigate the prognostic significance of LGI3 expression in NSCLC, gene expression microarray data of NSCLC patient cohorts were analyzed (Table III). The results revealed that the NSCLC studies demonstrated a significant association $(\mathrm{P}<0.05)$ of low LGI3 expression with poor prognosis of NSCLC (Table III, Fig. 4). Analysis of ADAM22 expression in the NSCLC cohorts revealed no consistent association between its expression and NSCLC prognosis (data not shown).

Somatic mutations of LGI3 in NSCLC. The somatic mutations of the LGI3 gene in NSCLC with amino acid alterations were identified in two major types of NSCLC, lung adenocarcinoma and lung squamous cell carcinoma (Table IV). A total of seven mutations with amino acid alterations were found in each NSCLC type, none of which occurred in both types of cancer. Venn diagram analysis of the amino acid variations in the five categories [conserved residues, phylogenetically coevolved residues, SNPs (12) and somatic mutations in two types of 

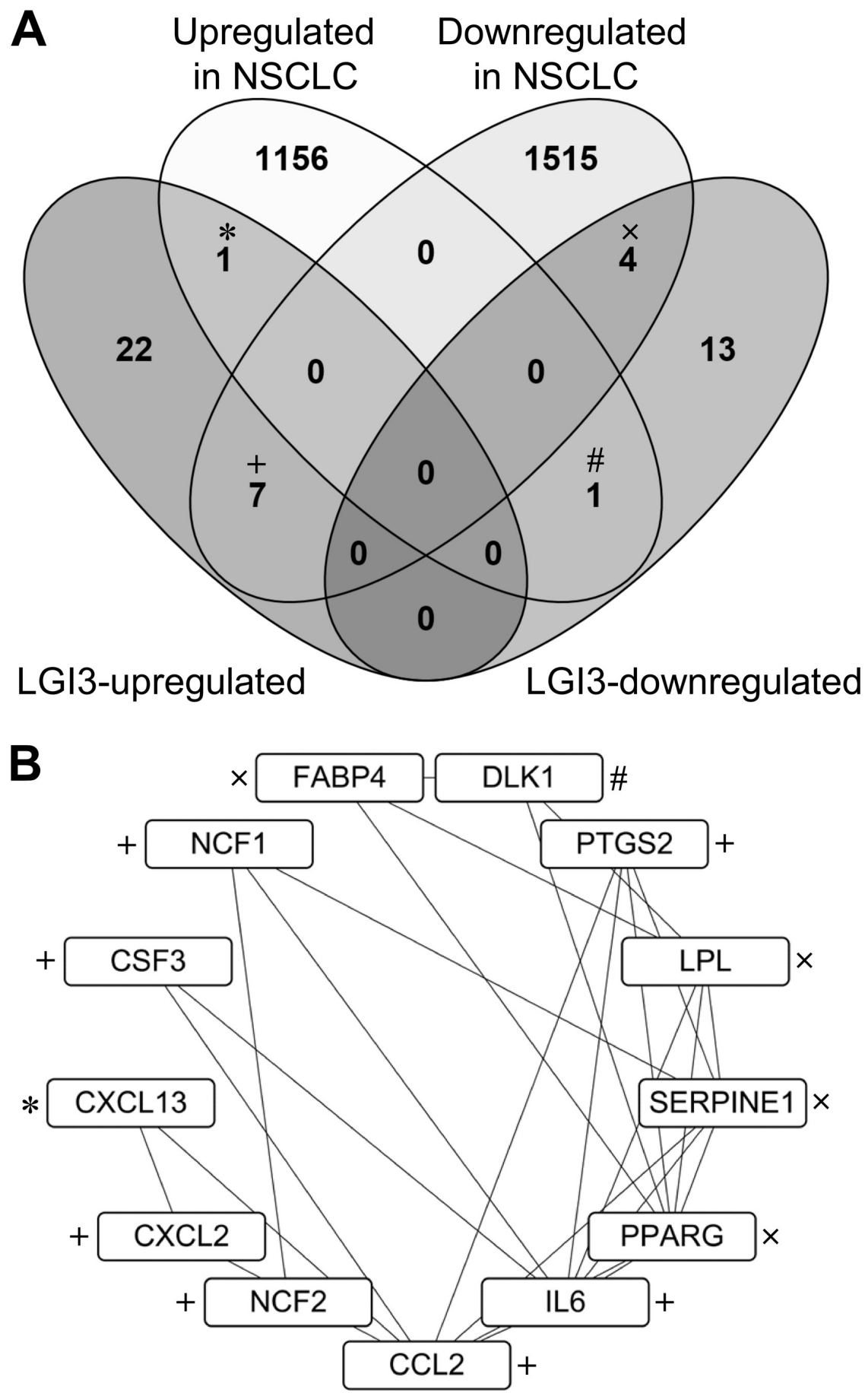

Figure 2. Comparative analysis of the up- and downregulated genes in NSCLC and LGI3-regulated genes. (A) Venn diagram showing the sets of the regulated gene categories. (B) Protein-protein interaction network of NSCLC-altered and LGI3-regulated products. The network includes nodes (gene products) and lines (pairwise protein interactions) sorted by interaction degrees. The symbols $\left({ }^{*},+,{ }^{+}\right.$and $\left.{ }^{\mathrm{x}}\right)$ indicate the gene products in the common sets of the regulated gene categories indicated in (A). CCL2, C-C motif chemokine ligand 2; CSF3, colony stimulating factor 3; CXCL, C-X-C motif chemokine ligand; DLK1, $\delta$ like non-canonical Notch ligand 1; FABP4, fatty acid binding protein 4; IL6, interleukin 6; LGI3, leucine rich repeat LGI family member 3; LPL, lipoprotein lipase; NCF, neutrophil cytosolic factor; NSCLC, non-small cell lung cancer; PPARG, peroxisome proliferator activated receptor $\gamma$; PTGS2, prostaglandin-endoperoxide synthase 2; SERPINE1, serpin family E member 1.

NSCLC] revealed that a subgroup of somatic mutation sites in NSCLC belonged to phylogenetically coevolved residues (Y293H in lung adenocarcinoma and A83V and L117F in lung squamous cell carcinoma) or SNP sites (S171stop and R430G in lung squamous cell carcinoma; Fig. 5). The amino acid alterations (S171stop and R430G) at SNP sites were different from the residues of the minor SNP alleles (Leu171, Cys430) and were not found in somatic mutations in NSCLC (12). No somatic mutation was identified at conserved residues.

\section{Discussion}

LGI protein members (LGI1, $-2,-3$ and -4 ) are differentially expressed in various tumor cells and LGI1 has been proposed 
Table I. Functional enrichment analysis of leucine rich repeat LGI family member 3-regulated genes that are altered in non-small cell lung cancer.

\begin{tabular}{llcl}
\hline Category & \multicolumn{1}{c}{ Term } & Count, n & P-value \\
\hline GOTERM_CC_DIRECT & GO:0005615 extracellular space & 8 & $1.37 \times 10^{-5}$ \\
GOTERM_BP_DIRECT & GO:0050729 positive regulation of inflammatory response & 4 & $2.19 \times 10^{-5}$ \\
GOTERM_CC_DIRECT & GO:0005576 extracellular region & 8 & $4.41 \times 10^{-5}$ \\
GOTERM_BP_DIRECT & GO:0071222 cellular response to lipopolysaccharide & 4 & $8.08 \times 10^{-5}$ \\
GOTERM_BP_DIRECT & GO:0006954 inflammatory response & 5 & $1.55 \times 10^{-4}$ \\
GOTERM_BP_DIRECT & GO:0006955 immune response & 5 & $2.33 \times 10^{-4}$ \\
GOTERM_BP_DIRECT & GO:0009409 response to cold & 3 & $3.43 \times 10^{-4}$ \\
GOTERM_MF_DIRECT & GO:0008009 chemokine activity & 3 & $6.31 \times 10^{-4}$ \\
GOTERM_BP_DIRECT & GO:0070098 chemokine-mediated signaling pathway & 3 & $1.33 \times 10^{-3}$ \\
GOTERM_BP_DIRECT & GO:0048010 vascular endothelial growth factor receptor & 3 & $1.37 \times 10^{-3}$ \\
& signaling pathway & 4 & $1.47 \times 10^{-3}$ \\
GOTERM_BP_DIRECT & GO:0042493 response to drug & 3 & $1.57 \times 10^{-3}$ \\
GOTERM_BP_DIRECT & GO:0045454 cell redox homeostasis & 3 & $3.16 \times 10^{-3}$ \\
GOTERM_BP_DIRECT & GO:0071356 cellular response to tumor necrosis factor & 3 & $4.45 \times 10^{-3}$ \\
GOTERM_BP_DIRECT & GO:0019221 cytokine-mediated signaling pathway & 3 & $6.50 \times 10^{-3}$ \\
GOTERM_MF_DIRECT & GO:0008201 heparin binding & 3 & $6.89 \times 10^{-3}$ \\
GOTERM_BP_DIRECT & GO:0032496 response to lipopolysaccharide & 3 & $1.24 \times 10^{-2}$ \\
GOTERM_BP_DIRECT & GO:0001525 angiogenesis & 3 & $2.62 \times 10^{-2}$ \\
GOTERM_MF_DIRECT & GO:0019899 enzyme binding & 3 & $2.92 \times 10^{-2}$ \\
GOTERM_MF_DIRECT & GO:0005102 receptor binding & 4 & $2.92 \times 10^{-2}$ \\
GOTERM_BP_DIRECT & GO:0007186 G-protein coupled receptor signaling pathway & 4 & $3.66 \times 10^{-2}$ \\
GOTERM_BP_DIRECT & GO:0045944 positive regulation of transcription from RNA & 3 & $4.23 \times 10^{-2}$ \\
GOTERM_BP_DIRECT & pol II promoter & GO:0045087 innate immune response & \\
\hline
\end{tabular}

BP, biological process; CC, cellular component; GO, Gene Ontology; MF, molecular function.

Table II. Kyoto Encyclopedia of Genes and Genomes pathway analysis of leucine rich repeat LGI family member 3-regulated genes that are altered in non-small cell lung cancer.

\begin{tabular}{lcc}
\hline Term & Count, $\mathrm{n}$ & P-value \\
\hline hsa04668: TNF signaling pathway & 4 & 0.00053 \\
hsa05144: Malaria & 3 & 0.00260 \\
hsa04062: Chemokine signaling & 4 & 0.00270 \\
pathway & & \\
hsa03320: PPAR signaling pathway & 3 & 0.00481 \\
hsa04060: Cytokine-cytokine & 4 & 0.00493 \\
receptor interaction & & \\
hsa05140: Leishmaniasis & 3 & 0.00539 \\
hsa05142: Chagas disease & 3 & 0.01129 \\
$\begin{array}{l}\text { (American trypanosomiasis) } \\
\text { hsa04380: Osteoclast }\end{array}$ & 3 & 0.01754 \\
differentiation & & \\
\hline
\end{tabular}

hsa, homo sapiens; NOD, nucleotide binding oligomerization; PPAR, peroxisome proliferator activated receptor; TNF, tumor necrosis factor. to be a tumor suppressor gene in brain tumors $(24,25)$. Our previous studies on LGI3 indicated that somatic mutations in various types of cancer were found in the LGI3 gene and subsets of the mutations affected SNP sites, phylogenetically coevolved amino acids and a conserved amino acid (12). Additionally, our previous studies demonstrated that the expression levels of LGI3 in tumor tissues are associated with the prognosis of brain, colorectal and lung cancers $(12,13)$. It was postulated that LGI3 may interact with the cytokine network in cancer through its genetic variations and dysregulated expression $(11,12)$.

The present study explored the potential prognostic value and functional network of LGI3 in NSCLC using integrative analysis of gene expression microarray data and the LGI3-regulated cytokine network $(11,12)$. Significant decreases in the expression levels of LGI3 and one of its receptors, ADAM22, in NSCLC tissues suggested that LGI3 may be involved in NSCLC carcinogenesis and progression via its receptor-mediated signaling pathway. Mediators of cellular signaling of LGI3 identified in our previous studies were: Akt and focal adhesion kinase (FAK) in LGI3-promoted neurite outgrowth (3), p53 and MDM2 in LGI3-promoted cell protection in ultraviolet B-irradiated keratinocytes (4), $\beta$-catenin and GSK3B in LGI3-induced keratinocyte migration (5), 

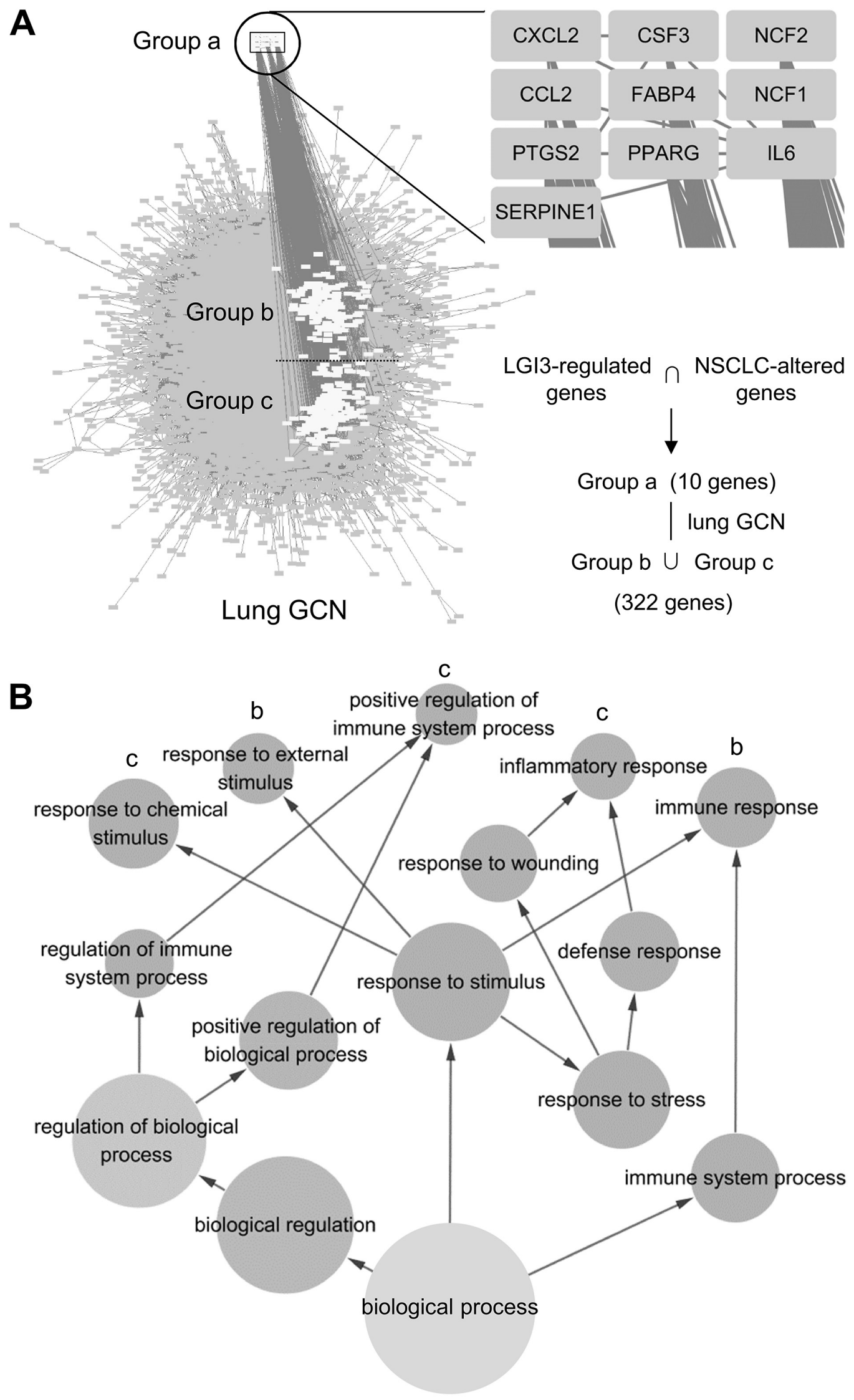

Figure 3. GCN analysis of NSCLC-altered and LGI3-regulated gene products in the lung. (A) NSCLC-altered and LGI3-regulated gene products found in the lung coexpression network (group a), and the subnetwork of the gene group (groups b and c) coexpressed with the genes in group a. (B) Gene Ontology category map of the subnetwork consisting of group $\mathrm{b}$ and $\mathrm{c}$ genes. Letters above the circles that represent leaf nodes of the hierarchical tree indicated the groups of the subnetworks in (A). CCL2, C-C motif chemokine ligand 2; CSF3, colony stimulating factor 3; CXCL2, C-X-C motif chemokine ligand 2; FABP4, fatty acid binding protein 4; GCN, gene coexpression network; IL6, interleukin 6; LGI3, leucine rich repeat LGI family member 3; NCF, neutrophil cytosolic factor; NSCLC, non-small cell lung cancer; PPARG, peroxisome proliferator activated receptor $\gamma$; PTGS2, prostaglandin-endoperoxide synthase 2; SERPINE1, serpin family E member 1. 
Table III. Dataset summary of expression microarray analyses for non-small cell lung cancer studies.

\begin{tabular}{lllccr}
\hline Group $^{\mathrm{a}}$ & Dataset & \multicolumn{1}{c}{ Array type } & Number of patients, $\mathrm{n}$ & Cut-off point & P-value \\
\hline A & GSE31210 & HG-U133_Plus_2 & 204 & 0.72 & 0.0098 \\
B & GSE8894 & HG-U133_Plus_2 & 138 & 0.22 & 0.0341 \\
C & GSE11117 & Novachip human 34.5k & 41 & 0.83 & 0.0247 \\
\hline
\end{tabular}

The groups correspond with the panels in Fig. 4.

A

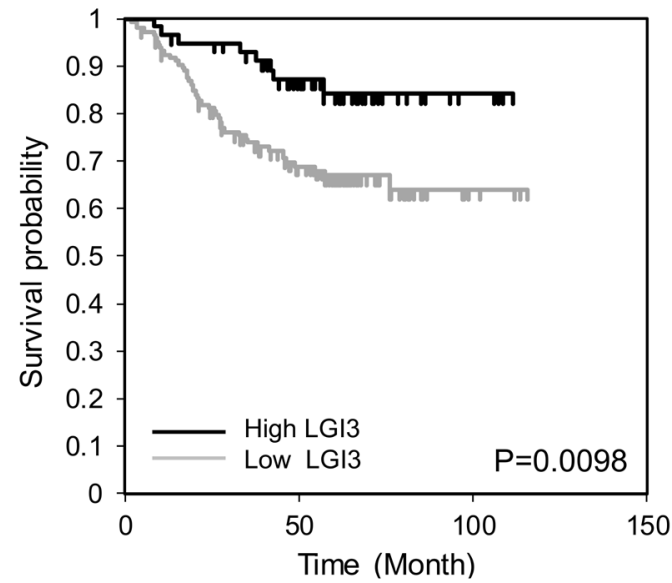

B

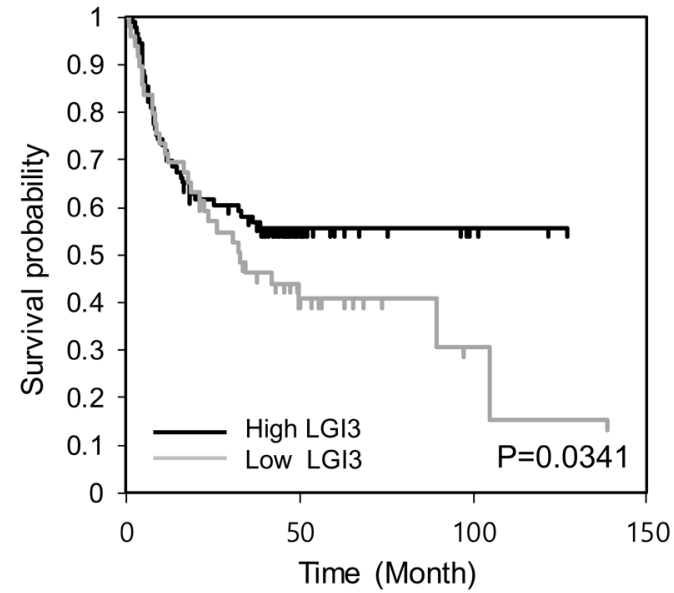

C

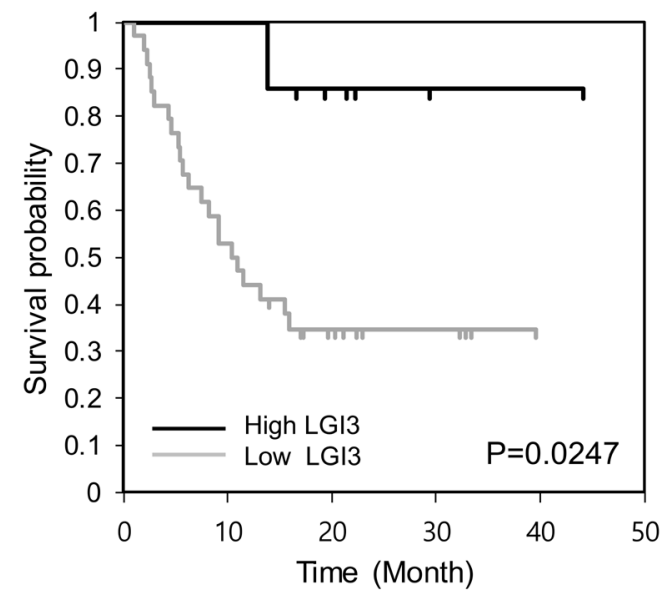

Figure 4. Association of LGI3 expression with the prognosis of patients with non-small cell lung cancer. Kaplan-Meier curves of the (A) GSE31210, (B) GSE8894 and (C) GSE11117 datasets of patient groups. LGI3, leucine rich repeat LGI family member 3.
MITF in melanogenesis (7), and PPARG, CCAAT-enhancer binding protein $\alpha$ and NF- $\mathrm{kB}$ in LGI3-regulated adipogenesis and metabolic inflammation $(9,10)$. Preadipocytes treated with LGI3 exhibit regulation of various signaling proteins (upregulated, Akt, AMP-activated protein kinase, Erk and PTEN; downregulated, eukaryotic translation initiation factor 4E binding protein 1, Bad and GSK3A) (11). The mediators of the LGI3-stimulated intracellular signaling pathway via ADAM22 should be addressed, and whether the signaling pathway is active and perturbed in NSCLC cells requires further investigation.

Our cumulative studies found multiple gene products that were regulated by LGI3 (1-5,7-11). A majority of the LGI3-regulated gene products (45/48 gene products) belong to a protein-protein interaction network that includes 16 cytokines, adipokines or chemokines, including ADIPOQ, CCL2/MCP-1, CSF1, CRP, CXCL2, CXCL13, CSF3, C5/HC, ESM1, IGF1, IGFBP1, IGFBP5, IL6, CCL12, TIMP1 and TNF- $\alpha$ (11). Among the 13 LGI3-regulated and NSCLC-altered gene products that formed a cluster of protein-protein interaction network, five gene products belonged to the cytokines or chemokines (CCL2, CSF3, CXCL2, CXCL13 and IL6). These results suggested that dysregulation of LGI3 may account for perturbation of the cytokine network and cell-cell communication in the microenvironment of NSCLC.

LGI3-regulated and NSCLC-altered genes were analyzed by functional enrichment and KEGG pathway analyses, and were determined to be significantly associated with the immune and inflammatory responses, chemokines and cytokine activities. These results were distinct from the same analyses of the LGI3-regulated genes that were altered in glioma in that the gene products were more significantly associated with angiogenesis, apoptosis, hypoxia, proliferation, $\mathrm{p} 53$ and hypoxia-inducible factor-1 signaling pathways in glioma (13). Therefore, LGI3 may be involved in the pathogenesis of NSCLC and glioma through common and distinctive mechanisms.

All LGI3-regulated and NSCLC-altered genes were found in the previous literature on NSCLC that reported an association of genetic variations, expression and function of these genes with NSCLC. Expression levels of PTGS2 (26), IL6 (27), CCL2 (28), NCF1/2 (29), CXCL2 (30), CSF3 (31), CXCL13 (32), LPL (33), SERPINE1 (34), PPARG (35) and FABP4 (36), and the promoter methylation of DLK1 (37) have been reported to be associated with the prognosis and pathogenesis of NSCLC. A total of seven genes (PTGS2, IL6, CCL2, NCF2, CXCL2, CSF3 and NCF1) that have been reported to be increased by LGI3 may be downregulated in 
Table IV. Somatic mutations of leucine rich repeat LGI family member 3 in NSCLC tissues.

\begin{tabular}{|c|c|c|c|c|c|c|c|}
\hline NSCLC type & Sample ID & $\begin{array}{l}\text { Protein } \\
\text { change }\end{array}$ & Mutation type & Chr 8 pos & Ref & Var & Allele freq $(\mathrm{T})$ \\
\hline Adenocarcinoma & LUAD-5V8LT & S531C & Missense & 22005729 & $\mathrm{~T}$ & A & NS \\
\hline Adenocarcinoma & LUAD-5V8LT & $\mathrm{P} 530 \mathrm{H}$ & Missense & 22005731 & $\mathrm{G}$ & $\mathrm{T}$ & NS \\
\hline Adenocarcinoma & TCGA-44-2656-01 & $\mathrm{E} 507^{\mathrm{a}}$ & Nonsense & 22005801 & $\mathrm{C}$ & A & 0.21 \\
\hline Adenocarcinoma & TCGA-91-6829-01 & Q460L & Missense & 22005941 & $\mathrm{~T}$ & A & 0.16 \\
\hline Adenocarcinoma & TCGA-55-A48X-01 & G82R & Missense & 22012939 & $\mathrm{C}$ & $\mathrm{T}$ & 0.09 \\
\hline Adenocarcinoma & TCGA-55-A490-01 & L185V & Missense & 22009455 & $\mathrm{G}$ & $\mathrm{C}$ & 0.40 \\
\hline Adenocarcinoma & TCGA-83-5908-01 & Y293H & Missense & 22006443 & A & $\mathrm{G}$ & 0.32 \\
\hline Squamous cell carcinoma & TCGA-66-2795-01 & L117F & Missense & 22012074 & $\mathrm{G}$ & A & 0.47 \\
\hline Squamous cell carcinoma & TCGA-52-7810-01 & $\mathrm{S} 171^{\mathrm{a}}$ & Nonsense & 22009496 & $\mathrm{G}$ & $\mathrm{C}$ & 0.51 \\
\hline Squamous cell carcinoma & TCGA-92-8065-01 & $\mathrm{E} 78 \mathrm{~K}$ & Missense & 22012951 & $\mathrm{C}$ & $\mathrm{T}$ & 0.24 \\
\hline Squamous cell carcinoma & TCGA-NC-A5HF-01 & $\mathrm{A} 83 \mathrm{~V}$ & Missense & 22012935 & $\mathrm{G}$ & A & 0.38 \\
\hline Squamous cell carcinoma & TCGA-85-8287-01 & A248V & Missense & 22009088 & $\mathrm{G}$ & A & 0.16 \\
\hline Squamous cell carcinoma & TCGA-85-8287-01 & $\mathrm{A} 248 \mathrm{P}$ & Missense & 22009089 & $\mathrm{C}$ & $\mathrm{G}$ & 0.17 \\
\hline Squamous cell carcinoma & TCGA-66-2785-01 & R430G & Missense & 22006032 & $\mathrm{G}$ & $\mathrm{C}$ & 0.25 \\
\hline
\end{tabular}

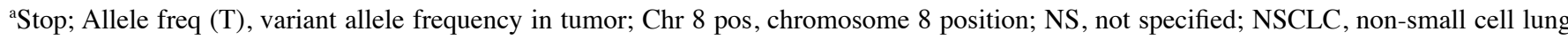
cancer; Ref, reference sequence; Var, variant sequence.

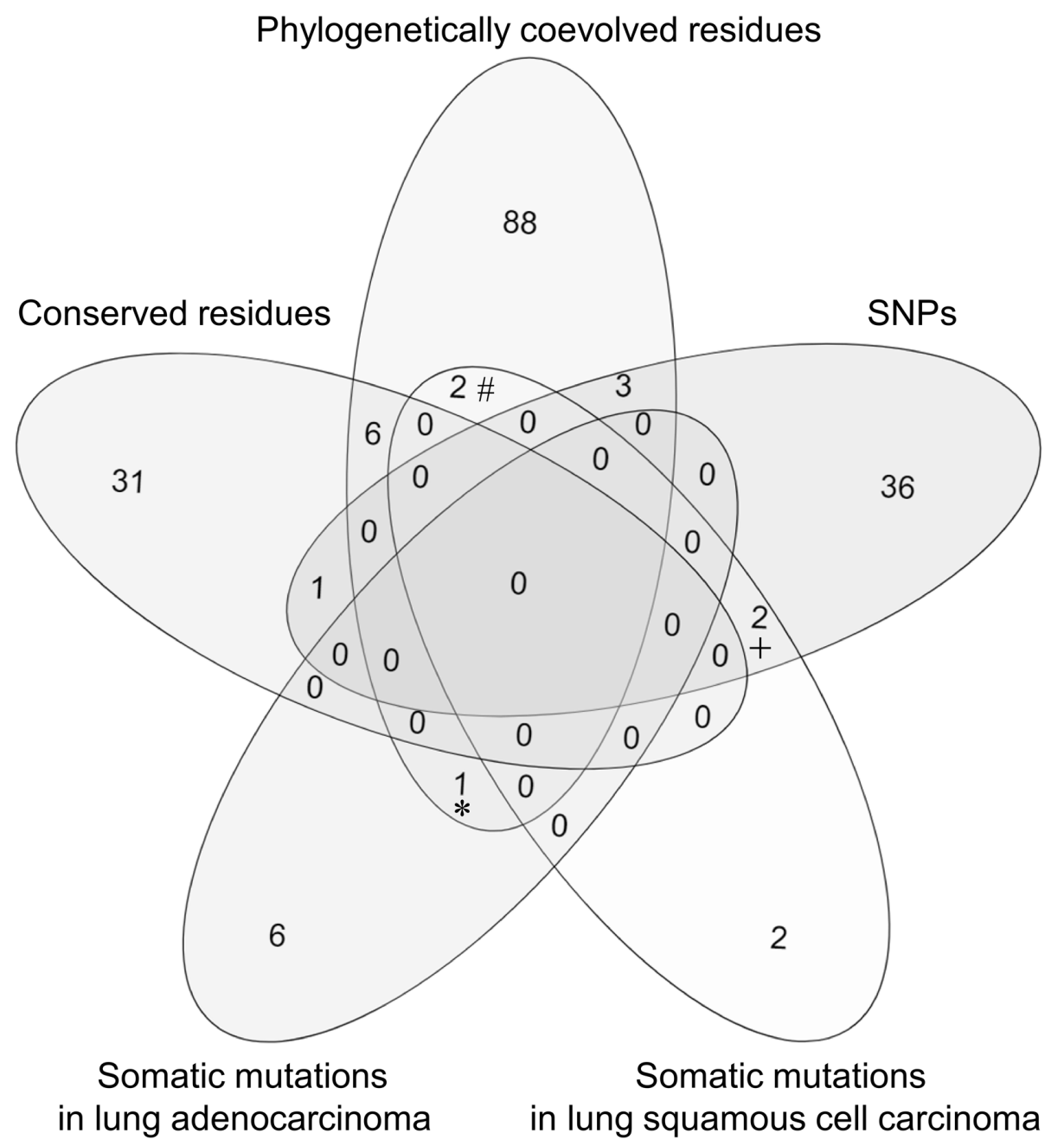

Figure 5. Comparative analysis of the somatic mutations in non-small cell lung cancer and genetic variations of leucine rich repeat LGI family member 3. The Venn diagram indicates the distribution of amino acid residues in the categories of variations. *Somatic mutations at phylogenetically coevolved amino acid residue 293. "Somatic mutations at phylogenetically coevolved amino acid residues 83 and 117 . ${ }^{+}$Somatic mutations at the amino acid residues with SNP sites (residues 171 and 430). SNP, single nucleotide polymorphism. 
NSCLC due to the suppression of LGI3 expression. DLK1, which has been revealed to be decreased by LGI3 (11), may be increased in NSCLC due to downregulation of LGI3. Thus, it may be postulated that the perturbation of the expression of the eight genes (PTGS2, IL6, CCL2, NCF2, CXCL2, CSF3, NCF1 and DLK1) was predominantly influenced by LGI3 downregulation in NSCLC. LGI3 may functionally interact with these gene products through protein-protein interaction networks in the implicated mechanisms as determined by the functional enrichment and KEGG pathway analyses.

LGI3 is abundantly expressed in the lung as well as diverse tissues, including the brain, skin, adipose tissues, liver, muscle, kidney and pancreas $(1,4,9,38)$. LGI3 has been demonstrated to be expressed in a variety of cell types, including neurons, keratinocytes, melanocytes, adipocytes and macrophages $(3,4,9,10)$. The RNA-sequencing data of the lung from the Human Protein Atlas (https://www.proteinatlas.org) indicates that LGI3 transcripts are distributed in pneumocytes $(20-40 \%$ of total transcripts per million), bronchial epithelium (5-10\%), endothelial cells (20-35\%) and macrophages (5-15\%). LGI3-expressing cell types in the mouse lung (mouse single cell transcriptome database; https://tabula-muris.ds.czbiohub.org) are notably similar to the human lung, supporting the validity of mouse models in studying LGI3 in human lung cancer. GCN analysis of LGI3-regulated and NSCLC-altered gene products in the present study further supported the notion that LGI3 is involved in NSCLC prognosis and pathogenesis as a regulatory cytokine in tumor immunity and inflammation. The gene products in the subnetwork (groups b and c) connected by gene coexpression with LGI3-regulated and NSCLC-altered genes, were demonstrated to be associated with common transcriptional regulatory processes of immune and inflammatory transcription factors [Elf-1 (39), Pea3 (40), Pu.1 (41), C-ets-1 (42), Usf1/2 (43), Stat6 (44), NF-kB (45), CREB (46), and AP-2 (47)]. Our previous study reported that NF- $\mathrm{KB}$ is a key transcription factor in mutual upregulation of LGI3 and TNF- $\alpha$, implicated in adipose tissue inflammation in obesity (10). Thus, downregulation of LGI3 in NSCLC may serve a role in the perturbation of the immune and inflammatory GCN of the lung, and may account for prognostic mechanisms of NSCLC.

The association of LGI3 expression with the prognosis of NSCLC identified in the present study suggests that LGI3 may serve antitumor roles in NSCLC progression. LGI3 has been demonstrated to stimulate intracellular signaling proteins and transcription factors involved in cancer, including p53, MDM2, Akt, $\beta$-catenin, FAK, NF- $\mathrm{BB}$ and MITF $(3,4,7,10,48-50)$. Dysregulation of these proteins by decreased LGI3 may be responsible for the prognostic role of LGI3 in NSCLC and glioma (13). Our previous study hypothesized that LGI3 may be a member of cytokine networks involved in obesity-associated metabolic disorders and cancer (11). Cytokine networks serve critical roles in anticancer immunity in the NSCLC microenvironment (51). By contrast, cytokine networks may promote tumor growth and metastasis through chronic inflammation (52). It was postulated that upregulated LGI3 in adipose tissue and plasma in obesity may promote chronic inflammation and cancer $(8,9,11)$. Cytokine perturbation in obesity may increase the risk of cancer in the digestive system, including the liver, pancreas and gastrointestinal tract (53). Consistent association between obesity and NSCLC has not been reported in previous studies; however, previous reports have suggested that being overweight is a positive prognostic factor $(54,55)$. Thus, the LGI3-regulated adipokine network in obesity may not account for the LGI3-regulated cytokine network in NSCLC prognosis $(8,10)$.

Our previous studies indicated that LGI3 increased M1-polarized macrophage markers (TNF- $\alpha$, inducible nitric oxide synthase, CCL2/MCP-1, CD11c and IL-6) (9-11). Macrophage polarization in the tumor microenvironment was investigated in NSCLC prognosis, and predominant M1 macrophages with inflammatory and antitumorigenic activities have been associated with a positive NSCLC prognosis (56). Thus, LGI3 may contribute to antitumor processes in the NSCLC microenvironment by promoting and maintaining M1-polarization of tumor-associated macrophages.

The somatic mutations of LGI3 in two major types of NSCLC suggested the potential prognostic value of the genetic variations of LGI3 in NSCLC. The mutations were distributed throughout the LGI3 protein domains (leucine-rich repeats and EPTP domains) and the residue 248 was the only site with multiple mutations (A248V and A248P). It was noted that two somatic mutations in lung squamous cell carcinoma (S171stop and R430G) were found at SNP sites with rare variants (global minor allele frequency, 0.0002) (12). These results warrant further studies on the prognostic and pathological roles of the genetic variations of LGI3 in NSCLC. Studies with LGI3-deficient or variant LGI3-expressing animal models and cell lines may provide further insight into the prognostic and pathological mechanisms of LGI3 in NSCLC.

In conclusion, the present study provided an integrative insight into the prognostic value of LGI3 in NSCLC by revealing the regulatory network of NSCLC-altered and LGI3-regulated gene products, and the association of expression and genetic variations of LGI3 with NSCLC. LGI3 may serve pathological as well as prognostic roles in NSCLC through its pro-inflammatory cytokine activity in immune and inflammatory processes of the tumor microenvironment.

\section{Acknowledgements}

Not applicable.

\section{Funding}

The present study was supported by Basic Science Research Program through the National Research Foundation of Korea (NRF) funded by the Ministry of Education (grant no. NRF-2 018R1D1A1A09082440).

\section{Availability of data and materials}

The datasets used and/or analyzed during the present study are available from the corresponding author on reasonable request.

\section{Authors' contributions}

HYY conceived and designed the study, performed data acquisition and analysis and wrote the manuscript. DSK and NSK contributed to the analysis and interpretation of data. All authors read and approved the final version. 


\section{Ethics approval and consent to participate}

Not applicable.

\section{Patient consent for publication}

Not applicable.

\section{Competing interests}

The authors declare that they have no competing interests.

\section{References}

1. Lee SE, Lee AY, Park WJ, Jun DH, Kwon NS, Baek KJ, Kim YG and Yun HY: Mouse LGI3 gene: Expression in brain and promoter analysis. Gene 372: 8-17, 2006.

2. Park WJ, Lee SE, Kwon NS, Baek KJ, Kim DS and Yun HY: Leucine-rich glioma inactivated 3 associates with syntaxin 1. Neurosci Lett 444: 240-244, 2008.

3. Park WJ, Lim YY, Kwon NS, Baek KJ, Kim DS and Yun HY: Leucine-rich glioma inactivated 3 induces neurite outgrowth through Akt and focal adhesion kinase. Neurochem Res 35 789-796, 2010

4. Lee SH, Jeong YM, Kim SY, Jeong HS, Park KC, Baek KJ, Kwon NS, Yun HY and Kim DS: Ultraviolet B-induced LGI3 secretion protects human keratinocytes. Exp Dermatol 21: 716-718, 2012

5. Jeong YM, Park WJ, Kim MK, Baek KJ, Kwon NS, Yun HY and Kim DS: Leucine-rich glioma inactivated 3 promotes $\mathrm{HaCaT}$ keratinocyte migration. Wound Repair Regen 21: 634-640, 2013

6. Kim IW, Jeong HS, Kwon NS, Baek KJ, Yun HY and Kim DS LGI3 promotes human keratinocyte differentiation via the Akt pathway. Exp Dermatol 27: 1224-1229, 2018.

7. Jeong HS, Jeong YM, Kim J, Lee SH, Choi HR, Park KC, Kim BJ, Baek KJ, Kwon NS, Yun HY and Kim DS: Leucine-rich glioma inactivated 3 is a melanogenic cytokine in human skin. Exp Dermatol 23: 600-602, 2014.

8. Kim HA, Kwon NS, Baek KJ, Kim DS and Yun HY: Leucine-rich glioma inactivated 3 associates negatively with adiponectin. Cytokine 62: 206-209, 2013

9. Kim HA, Park WJ, Jeong HS, Lee HE, Lee SH, Kwon NS, Baek KJ, Kim DS and Yun HY: Leucine-rich glioma inactivated 3 regulates adipogenesis through ADAM23. Biochim Biophys Acta 1821: 914-922, 2012

10. Kim HA, Kwon NS, Baek KJ, Kim DS and Yun HY: Leucine-rich glioma inactivated 3 and tumor necrosis factor- $\alpha$ regulate mutually through NF- $\kappa$ B. Cytokine 72: 220-223, 2015.

11. Kim HA, Kwon NS, Baek KJ, Kim DS and Yun HY: Leucine-rich glioma inactivated 3: Integrative analyses support its role in the cytokine network. Int J Mol Med 40: 251-259, 2017.

12. Kwon NS, Baek KJ, Kim DS and Yun HY: Leucine-rich glioma inactivated 3: Integrative analyses reveal its potential prognostic role in cancer. Mol Med Rep 17: 3993-4002, 2018.

13. Kwon NS, Kim DS and Yun HY: Leucine-rich glioma inactivated 3: Integrative analyses support its prognostic role in glioma. Onco Targets Ther 10: 2721-2728, 2017

14. Herbst RS, Morgensztern D and Boshoff C: The biology and management of non-small cell lung cancer. Nature 553: 446-454, 2018.

15. Lu TP, Tsai MH, Lee JM, Hsu CP, Chen PC, Lin CW, Shih JY, Yang PC, Hsiao CK, Lai LC and Chuang EY: Identification of a novel biomarker, SEMA5A, for non-small cell lung carcinoma in nonsmoking women. Cancer Epidemiol Biomarkers Prev 19: 2590-2597, 2010.

16. Gautier L, Cope L, Bolstad BM and Irizarry RA: affy-analysis of Affymetrix GeneChip data at the probe level. Bioinformatics 20 : 307-315, 2004

17. Szklarczyk D, Franceschini A, Wyder S, Forslund K, Heller D, Huerta-Cepas J, Simonovic M, Roth A, Santos A, Tsafou KP, et al STRING v10: Protein-protein interaction networks, integrated over the tree of life. Nucleic Acids Res 43 (Database Issue) D447-D452, 2015.

18. Lopes CT, Franz M, Kazi F, Donaldson SL, Morris Q and Bader GD: Cytoscape Web: An interactive web-based network browser. Bioinformatics 26: 2347-2348, 2010.
19. Huang da W, Sherman BT and Lempicki RA: Systematic and integrative analysis of large gene lists using DAVID bioinformatics resources. Nat Protoc 4: 44-57, 2009.

20. Lee S, Zhang C, Liu Z, Klevstig M, Mukhopadhyay B, Bergentall M, Cinar R, Ståhlman M, Sikanic N, Park JK, et al: Network analyses identify liver-specific targets for treating liver diseases. Mol Syst Biol 13: 938, 2017.

21. Thomas-Chollier M, Hufton A, Heinig M, Heinig M, O'Keeffe S, Masri NE, Roider HG, Manke T and Vingron M: Transcription factor binding predictions using TRAP for the analysis of ChIP-seq data and regulatory SNPs. Nat Protoc 6: 1860-1869, 2011.

22. Mizuno H, Kitada K, Nakai K and Sarai A: PrognoScan: A new database for meta-analysis of the prognostic value of genes. BMC Med Genomics 2: 18, 2009

23. Li H, Sun Y and Zhan M: Exploring pathways from gene co-expression to network dynamics. Methods Mol Biol 541: 249-267, 2009

24. Rossi MR, Huntoon K and Cowell JK: Differential expression of the LGI and SLIT families of genes in human cancer cells. Gene 356: 85-90, 2005.

25. Chernova OB, Somerville RP and Cowell JK: A novel gene, LGI1, from 10q24 is rearranged and downregulated in malignant brain tumors. Oncogene 17: 2873-2881, 1998.

26. Pan J, Yang Q, Shao J, Zhang L, Ma J, Wang Y, Jiang BH, Leng $J$ and Bai $X$ : Cyclooxygenase-2 induced $\beta 1$-integrin expression in NSCLC and promoted cell invasion via the EP1/MAPK/E2F-1/FoxC2 signal pathway. Sci Rep 6: 33823, 2016.

27. Shintani Y, Fujiwara A, Kimura T, Kawamura T, Funaki S, Minami M and Okumura M: IL-6 secreted from cancer-associated fibroblasts mediates chemoresistance in NSCLC by increasing epithelial-mesenchymal transition signaling. J Thorac Oncol 11: 1482-1492, 2016.

28. Li L, Liu YD, Zhan YT, Zhu YH, Li Y, Xie D and Guan XY: High levels of CCL2 or CCL4 in the tumor microenvironment predict unfavorable survival in lung adenocarcinoma. Thorac Cancer 9: 775-784, 2018.

29. Song NY, Zhu F, Wang Z, Willette-Brown J, Xi S, Sun Z, Su L, $\mathrm{Wu} \mathrm{X}, \mathrm{Ma} \mathrm{B}$, Nussinov R, et al: IKK $\alpha$ inactivation promotes Kras-initiated lung adenocarcinoma development through disrupting major redox regulatory pathways. Proc Natl Acad Sci USA 115: E812-E821, 2018.

30. Matsuo N, Azuma K, Hattori S, Ohtake J, Kawahara A, Ishii H, Tokito T, Yamada K, Shibata Y, Shimokawaji T, et al: Association between soluble immune mediators and tumor responses in patients with non-small cell lung cancer treated with anti-PD-1 inhibitor. Int J Cancer 144: 1170-1179, 2019.

31. Tan X and Chen M: MYLK and MYL9 expression in non-small cell lung cancer identified by bioinformatics analysis of public expression data. Tumour Biol 35: 12189-12200, 2014

32. Eide HA, Halvorsen AR, Sandhu V,Fåne A, Berg J,Haakensen VD, Kure EH, Brustugun OT, Kiserud CE, Kyte JA and Helland ̊: Non-small cell lung cancer is characterised by a distinct inflammatory signature in serum compared with chronic obstructive pulmonary disease. Clin Transl Immunology 5: e109, 2016.

33. Podgornik H, Sok M, Kern I, Marc J and Cerne D: Lipoprotein lipase in non-small cell lung cancer tissue is highly expressed in a subpopulation of tumor-associated macrophages. Pathol Res Pract 209: 516-520, 2013

34. Eberlein C, Rooney C, Ross SJ, Farren M, Weir HM and Barry ST: E-Cadherin and EpCAM expression by NSCLC tumour cells associate with normal fibroblast activation through a pathway initiated by integrin $\alpha v \beta 6$ and maintained through TGF $\beta$ signalling. Oncogene 34: 704-716, 2015.

35. Bren-Mattison Y, Van Putten V, Chan D, Winn R, Geraci MW and NemenoffRA:Peroxisome proliferator-activated receptor-gamma (PPAR(gamma)) inhibits tumorigenesis by reversing the undifferentiated phenotype of metastatic non-small-cell lung cancer cells (NSCLC). Oncogene 24: 1412-1422, 2005.

36. Tang Z, Shen Q, Xie H, Zhou X, Li J, Feng J, Liu H, Wang W, Zhang S and Ni S: Elevated expression of FABP3 and FABP4 cooperatively correlates with poor prognosis in non-small cell lung cancer (NSCLC). Oncotarget 7: 46253-46262, 2016.

37. Zhong Z, Ye Y, Guo W, He Y and Hu W: Relationship between DLK1 gene promoter region DNA methylation and non-small cell lung cancer biological behavior. Oncol Lett 13: 4123-4126, 2017.

38. Gu W, Wevers A, Schröder H, Grzeschik KH, Derst C, Brodtkorb E, de Vos R and Steinlein OK: The LGI1 gene involved in lateral temporal lobe epilepsy belongs to a new subfamily of leucine-rich repeat proteins. FEBS Lett 519: 71-76, 2002. 
39. Kumazoe M, Yamashita M, Nakamura Y, Takamatsu K, Bae J, Yamashita S, Yamada S, Onda H, Nojiri T, Kangawa K and Tachibana H: Green tea polyphenol EGCG upregulates Tollip expression by suppressing Elf-1 expression. J Immunol 199: 3261-3269, 2017.

40. Kramer B, Wiegmann K and Kronke M: Regulation of the human TNF promoter by the transcription factor Ets. J Biol Chem 270: 6577-6583, 1995.

41. DeKoter RP and Singh H: Regulation of B lymphocyte and macrophage development by graded expression of PU.1. Science 288: 1439-1441, 2000.

42. Bevington SL, Cauchy P, Piper J, Bertrand E, Lalli N, Jarvis RC, Gilding LN, Ott S, Bonifer C and Cockerill PN: Inducible chromatin priming is associated with the establishment of immunological memory in T cells. EMBO J 35: 515-535, 2016.

43. Corre $\mathrm{S}$ and Galibert MD: USF as a key regulatory element of gene expression. Med Sci (Paris) 22: 62-67, 2006 (In French).

44. Swain SD, Meissner NN, Siemsen DW, McInnerney K and Harmsen AG: Pneumocystis elicits a STAT6-dependent, strain-specific innate immune response and airway hyperresponsiveness. Am J Respir Cell Mol Biol 46: 290-298, 2012.

45. Zhang Q, Lenardo MJ and Baltimore D: 30 Years of NF- $\kappa B$ : A blossoming of relevance to human pathobiology. Cell 168: 37-57, 2017.

46. Wen AY, Sakamoto KM and Miller LS: The role of the transcription factor CREB in immune function. J Immunol 185: 6413-6419, 2010.

47. Oyama N, Iwatsuki K, Homma Y and Kaneko F: Induction of transcription factor AP-2 by inflammatory cytokines in human keratinocytes. J Invest Dermatol 113: 600-606, 1999.
48. Ji Z, Erin CY, Kumar R, Taylor M, Jenny Njauw CN, Miao B, Frederick DT, Wargo JA, Flaherty KT, Jönsson G and Tsao H: MITF modulates therapeutic resistance through EGFR signaling. J Invest Dermatol 135: 1863-1872, 2015.

49. Sulzmaier FJ, Jean C and Schlaepfer DD: FAK in cancer: Mechanistic findings and clinical applications. Nat Rev Cancer 14: 598-610, 2014.

50. Uzdensky AB, Demyanenko SV and Bibov MY: Signal transduction in human cutaneous melanoma and target drugs. Curr Cancer Drug Targets 13: 843-866, 2013.

51. Domagala-Kulawik J: The role of the immune system in non-small cell lung carcinoma and potential for therapeutic intervention. Transl Lung Cancer Res 4: 177-190, 2015.

52. West NR, McCuaig S, Franchini F and Powrie F: Emerging cytokine networks in colorectal cancer. Nat Rev Immunol 15: 615-629, 2015.

53. Font-Burgada J, Sun B and Karin M: Obesity and cancer: The oil that feeds the flame. Cell Metab 23: 48-62, 2016

54. Lam VK, Bentzen SM, Mohindra P, Nichols EM, Bhooshan N, Vyfhuis M, Scilla KA, Feigenberg SJ, Edelman MJ and Feliciano JL: Obesity is associated with long-term improved survival in definitively treated locally advanced non-small cell lung cancer (NSCLC). Lung Cancer 104: 52-57, 2017.

55. Xie HJ, Zhang X, Wei ZQ, Long H, Rong TH and Su XD: Effect of body mass index on survival of patients with stage I non-small cell lung cancer. Chin J Cancer 36: 7, 2017.

56. Jackute J, Zemaitis M, Pranys D, Sitkauskiene B, Miliauskas S, Vaitkiene S and Sakalauskas R: Distribution of M1 and M2 macrophages in tumor islets and stroma in relation to prognosis of non-small cell lung cancer. BMC Immunol 19: 3, 2018. 